This paper has been published in Bulletin of the Australian Mathematical Society, 86(1):22-28 (2012).

Copyright 2012 by Australian Mathematical Publishing Association Inc and Cambridge University Press Journals.

The final publication is available at

http://dx.doi.org/10.1017/S0004972711003418

http://journals. cambridge.org/abstract_S0004972711003418 


\title{
SOME SOLUBILITY CRITERIA IN FACTORISED GROUPS
}

\author{
M. ASAAD, A. BALLESTER-BOLINCHES ${ }^{\bowtie}$ and R. ESTEBAN-ROMERO \\ Dedicated to John Cossey on the occasion of his 70th birthday
}

\begin{abstract}
In this paper, solubility of groups factorised as a product of two subgroups which are connected by certain permutability properties is studied.

2010 Mathematics subject classification: primary 20D10.

Keywords and phrases: factorised group, mutually m-permutable product, soluble group.
\end{abstract}

\section{Introduction and statement of results}

All groups considered in this paper are finite.

Groups which are factorised as a product of two subgroups have been the subject of many investigations in group theory. Let the group $G=A B$ be the product of two subgroups $A$ and $B$. It is rather clear that not many properties carry over from $A$ and $B$ to $G$, and so a natural problem is: Suppose that $A$ and $B$ belong to a certain class of groups, what can be said about $G$ ? This question has been extensively studied with many results available spread over many papers (see [1,3]). The fact that the product of two supersoluble groups is not supersoluble in general, even when both factors are normal, has open the door to the study of groups which are factorised as a product of two subgroups connected by some permutability properties. The cases when $A$ and $B$ are totally permutable, that is, every subgroup of $A$ permutes with every subgroup of $B$, or $A$ and $B$ are mutually permutable, that is, every subgroup of $A$ permutes with $B$ and every subgroup of $B$ permutes with $A$, produced many surprising and nice results (see [3]).

Sometimes it is interesting to impose permutability of one factor not with the family of all subgroups of the other, but with a smaller relevant family of subgroups, for instance, the case of the family of all maximal subgroups. Then mutually $\mathrm{m}$ permutable products, that is, groups $G=A B$ factorised as a product of two subgroups $A$ and $B$ such that $A$ permutes with every maximal subgroup of $B$ and $B$ permutes with every maximal subgroup of $A$, appear. A first example of these products was studied 
by Maier [9], who studied subgroups $H$ of a group $G$ which permute with all maximal subgroups of $G$. In this case, $G$ is the mutually m-permutable product of $H$ and $G$. The second author, Guo, and Pedraza-Aguilera have studied in [4] the behaviour of mutually m-permutable products with respect to saturated formations containing the class of all supersoluble groups:

Theorem 1.1 ([4, Theorems 1 and 2]). Let the soluble group $G=G_{1} G_{2} \cdots G_{r}$ be the product of the pairwise mutually m-permutable subgroups $G_{1}, G_{2}, \ldots, G_{r}$. Suppose also that $G^{\prime}$ is nilpotent.

1. Suppose that $\mathfrak{F}$ is a saturated formation containing the class $\mathfrak{U}$ of all supersoluble groups. If $G_{i}$ is an $\mathfrak{F}$-group for each $i \in\{1,2, \ldots, r\}$, then $G$ belongs to $\mathfrak{F}$.

2. Suppose that $\mathfrak{F}$ is a saturated formation and that $G$ is an $\mathfrak{F}$-group. Then $G_{i} \in \mathfrak{F}$ for all $i \in\{1,2, \ldots, r\}$.

The second author, Cossey, and Pedraza-Aguilera have proved the following result:

Theorem 1.2 ([2, Theorem 2]). Let the group $G=A B$ be the mutually m-permutable product of its subgroups $A$ and $B$. If $A$ and $B$ are supersoluble, then $G$ is soluble.

Ezquerro and Soler-Escrivà have extended Theorem 1.2 to mutually m-permutable products of soluble groups.

Theorem 1.3 ([7, Theorem 1.2]). Let the group $G=A B$ be the product of the mutually m-permutable subgroups $A$ and $B$. If $A$ and $B$ are soluble, then $G$ is soluble.

The main aim of this paper is to analyse the solubility of factorised groups whose factors are connected by more general permutability conditions. Our first result can be seen as a generalisation of Theorem 1.3 for factors of coprime orders.

Theorem 1.4. Assume that a group $G=A B$ is the product of the soluble subgroups $A$ and $B$ and let $p$ be the smallest prime dividing the order of $G$. If $\operatorname{gcd}(|A|,|B|)=1, p$ divides the order of $A$, and $B$ permutes with every maximal subgroup of $A$, then $G$ is soluble.

The following result can be regarded as a generalisation of Theorem 1.2 in which permutability is required only for the maximal subgroups whose index is not divisible by the smallest prime dividing the order of the group.

Theorem 1.5. Let the group $G=A B$ be the product of the supersoluble subgroups $A$ and $B$ and let $p$ be the smallest prime dividing the order of $G$. If $A$ permutes with every maximal subgroup $B_{1}$ of $B$ such that $p$ does not divide $\left|B: B_{1}\right|$ and $B$ permutes with every maximal subgroup $A_{1}$ of $A$ such that $p$ does not divide $\left|A: A_{1}\right|$, then $G$ is soluble.

Finally, if in Theorem 1.5 one of the factors, $B$ say, is nilpotent, the conclusion is achieved without requiring $A$ to permute with the maximal subgroups of $B$ whose index is not divisible by $p$. 
Theorem 1.6. Let the group $G=A B$ be the product of the subgroups $A$ and $B$ and let $p$ be the smallest prime dividing the order of $G$. Assume that $A$ is supersoluble and $B$ is nilpotent. If $B$ permutes with every maximal subgroup $A_{1}$ of $A$ such that $p$ does not divide the index $\left|A: A_{1}\right|$, then $G$ is soluble.

The notation is standard and can be found in books like [6] or[8].

\section{Preliminary results}

Let $p$ be a prime number and let $G$ be a group. Deskins ([5], see also [8, III, Aufgabe 12]) defined the subgroup $\Phi_{p}(G)$ as the intersection of all maximal subgroups of $G$ with index not divisible by $p$. He proved:

Theorem 2.1. Let $p$ be a prime number and let $G$ be a group. Then $\Phi_{p}(G)$ has a normal Sylow p-subgroup $P$ such that $\Phi_{p}(G) / P$ is nilpotent.

The following results of Ezquerro and Soler-Escrivà are needed in our proofs. The first one follows from the arguments contained in the proof of [7, Lemma 2.2].

Lemma 2.2. Let the group $G=A B$ be the product of its subgroups $A$ and $B$ and let $D=A \cap B$. Assume that $B$ permutes with each maximal subgroup $A_{1}$ of $A$ such that $D \leq A_{1}$. Then

$\left(\bigcap\left\{A_{1} \mid A_{1}\right.\right.$ maximal in $\left.\left.A, D \leq A_{1}\right\}\right) B=\bigcap\left\{B A_{1} \mid A_{1}\right.$ maximal in $\left.A, D \leq A_{1}\right\}$ is a subgroup of $G$.

The second one is useful in proofs by minimal counterexample.

Lemma 2.3 ([7, Lemma 2.3]). Let $G$ be a primitive group with a unique minimal normal subgroup and suppose that $G=A B$, where $A$ and $B$ are non-trivial subgroups of $G$ such that $B$ permutes with all maximal subgroups of $A$. Let $A_{1}$ be a maximal subgroup of $A$ such that $B A_{1}$ is a core-free maximal subgroup of $G$. Then:

1. $\quad H$ is a Sylow p-subgroup of $G$ of order $p$, where $p$ is the greatest prime dividing $|G|$, and

2. $B$ is a core-free maximal subgroup of $G$ such that $|G: B|=p$.

The following result was proved by Maier.

Lemma 2.4 ([9, Lemma 2.10]). Let $G$ be a group and let $X$ be a subgroup of $G$. If $X$ permutes with all maximal subgroups of $G$ whose index is coprime with the order of the Fitting subgroup $\mathrm{F}(X)$, then $\mathrm{F}(X) \leq \mathrm{F}(G)$.

Our proof of Theorem 1.5 is based on the following lemma.

Lemma 2.5. Let the group $G=A B$ be the product of its non-trivial subgroups $A$ and $B$ and suppose that $A \cap B=1$. Assume that $G$ has a unique minimal normal subgroup, $N$ say. If $A_{1}$ is a maximal subgroup of $A$ such that $B$ permutes with $A_{1}^{a}$ for all $a \in A$ and $\operatorname{Core}_{G}\left(A_{1} B\right)=1$, then $A$ has prime order or $N \leq A$. 
Proof. We prove first that $B$ centralises $A_{1}$. By hypothesis $A_{1}^{a} B$ is a subgroup of $G$ for each $a \in A$. Consider $g \in G$, then $g=a b$, where $a \in A$ and $b \in B$. Hence $\left(A_{1}^{a} B\right)^{b}=A_{1}^{a b} B^{b}=A_{1}^{g} B \leq G$. This implies that $B$ permutes with $A_{1}^{g}$ for every $g \in G$. By $[1,2.5 .1]$ we have that $\left[B, A_{1}\right]$ is subnormal in $G$. Hence $N$ normalises $\left[B, A_{1}\right]$ by [1, 7.5.5]. On the other hand, since $A \cap B=1, A_{1} B$ is a maximal subgroup of $G$ and $\operatorname{Core}_{G}\left(A_{1} B\right)=1$. Therefore $G=\left(A_{1} B\right) N$ and $\left[B, A_{1}\right]$ is a normal subgroup of $G$ contained in $A_{1} B$. Thus $\left[B, A_{1}\right]=1$ and $B$ centralises $A_{1}$. Consider now the normal closure $\left\langle A_{1}^{G}\right\rangle$ of $A_{1}$ in $G$, which is contained in $A$. Then $A_{1}^{G}=A_{1}$ or $\left\langle A_{1}^{G}\right\rangle=A$ and so $A$ has prime order or $N \leq A_{1}^{G}=A$.

\section{Proofs of the main theorems}

Proof of Theorem 1.4. Suppose that the result is not true. Let $G$ be a counterexample of minimal order. We may assume $p=2$, otherwise by the well-known Feit-Thompson theorem, $G$ would be soluble, which would contradict the choice of $G$. Let $N$ be a minimal normal subgroup of $G$. If 2 divides the order of $A N / N$, the minimal choice of $G$ implies that $G / N$ is soluble. Moreover if 2 does not divide the order of $A N / N$, then 2 does not divide the order of $G / N$ and as above $G / N$ is soluble. Thus any proper quotient group of $G$ is soluble. Since the class of all soluble groups is a saturated formation, it follows that $\Phi(G)=1$ and $G$ has a unique minimal normal subgroup $N=\operatorname{Soc}(G), N$ is non-abelian, and $\mathrm{C}_{G}(N)=1$.

On the other hand, we may suppose $|A| \neq 2$ because otherwise $G$ would be 2-nilpotent and hence soluble, a contradiction to the choice of $G$. Let $A_{1}$ be a maximal subgroup of $A$. Then, by hypothesis, $A_{1} B$ is a maximal subgroup of $G$. If Core $_{G}\left(A_{1} B\right)=1$, the application of Lemma 2.3 yields that $A$ is a Sylow $p$-subgroup of $G$ of order $p$ for the largest prime $p$ dividing $|G|$. This contradicts the hypothesis that 2 divides $|A|$. Therefore $\operatorname{Core}_{G}\left(A_{1} B\right) \neq 1$ for every maximal subgroup $A_{1}$ of $A$. Consequently $N$ is contained in $A_{1} B$ for every maximal subgroup $A_{1}$ of $A$. By Lemma $2.2, N \leq B \Phi(A)$ and so $A \cap N \leq A \cap B \Phi(A)=\Phi(A)$. Let $\pi$ denote the set of primes dividing $|A \cap N|$. Since $\operatorname{gcd}(|A \cap N|,|G: A|)=1$, by [8, IV, 4.6] there exists a normal subgroup $R$ of $N$ such that $N / R$ is a $\pi$-group, $R \cap A=1$, and $|N \cap A|$ divides $|N: R|$. Moreover, $|N: A \cap N|$ divides $|G: A|$, which is a $\pi^{\prime}$-number. Hence $N=R(A \cap N)$. On the other hand, since $\operatorname{gcd}(|A|,|B|)=1$, it follows that $N \cap A$ is a Hall $\pi$-subgroup of $N$ and $N \cap B$ is a Hall $\pi^{\prime}$-subgroup of $N$. Thus $N=(N \cap A)(N \cap B)$. By order considerations, bearing in mind that $R \cap A=1$, we obtain that $|R|=|N \cap B|$. Now $R$ is a normal $\pi^{\prime}$-subgroup of $N$ and $N \cap B$ is a Hall $\pi^{\prime}$-subgroup of $N$, so $R=N \cap B$. But then $R$ and $N / R \cong N \cap A$ are soluble. This leads to the solubility of $N$, the final contradiction which completes the proof of the theorem.

Proof of Theorem 1.5. Assume that the result is not true and let $G$ be a minimal counterexample. Let $1 \neq N$ be a normal subgroup of $G$. If $p$ does not divide $|G / N|$, then, by hypothesis, $A N / N$ permutes with every maximal subgroup of $B N / N$ and $B N / N$ permutes with every maximal subgroup of $A N / N$. Applying Theorem 1.2, we have that $G / N$ is soluble. On the other hand, if $p$ divides the order of $G / N$, the 
hypotheses of the theorem hold in $G / N$. Therefore the minimal choice of $G$ implies that $G / N$ is soluble. Consequently $\operatorname{Soc}(G)=N$ is the unique minimal normal subgroup of $G, N$ is non-abelian, and $\mathrm{C}_{G}(N)=1$.

By the fact that $B$ permutes with every maximal subgroup $A_{1}$ of $A$ such that $p$ does not divide $\left|A: A_{1}\right|$, it follows that $A \cap B$ permutes with every maximal subgroup $A_{1}$ of $A$ such that $p$ does not divide $\left|A: A_{1}\right|$. Assume that $A_{1}$ is a maximal subgroup of $A$ such that $p$ divides $\left|A: A_{1}\right|$. Since $A$ is supersoluble, it is clear that $\left|A: A_{1}\right|=p$. Moreover, the fact that $p$ is the smallest prime dividing the order of $G$ yields that $A_{1}$ is a normal subgroup of $A$. Consequently $A \cap B$ permutes with $A_{1}$ and so $A \cap B$ permutes with all maximal subgroups of $A$. With the same arguments, $A \cap B$ permutes with every maximal subgroup of $B$ of index $p$. By Lemma 2.4, $\mathrm{F}(A \cap B) \leq \mathrm{F}(A)$ and $\mathrm{F}(A \cap B) \leq \mathrm{F}(B)$. Thus $\mathrm{F}(A \cap B)$ is a subnormal subgroup of $A$ and $B$, respectively. Now an application of $[1,7.7 .1]$ yields that $\mathrm{F}(A \cap B)$ is a subnormal subgroup of $G$. Moreover $\mathrm{F}(A \cap B)$ is nilpotent. Consequently, $\mathrm{F}(A \cap B) \leq \mathrm{F}(G)=1$. Hence the solubility of $A \cap B$ implies that $A \cap B=1$.

We will prove now that $p$ divides the orders of $A$ and $B$. It is clear that we may assume that $p$ divides $|A|$. Assume that $p$ does not divide $|B|$. Thus, by hypothesis, $A$ permutes with every maximal subgroup of $B$. We may suppose that $A$ is not nilpotent, otherwise $G$ is soluble by [2, Theorem 1]. Let $B_{1}$ be a maximal subgroup of $B$. Then $A B_{1}$ is a maximal subgroup of $G$.

Assume that $\operatorname{Core}_{G}\left(A B_{1}\right)=1$. By Lemma 2.5, $N \leq B$, which contradicts the solubility of $B$, or $B$ has prime order. Suppose $|B|=q$, where $q \neq p$ is a prime number. Then $|G: A|=q$ and $\operatorname{Core}_{G}(A)=1$. This means that $G$ is isomorphic to a subgroup of the symmetric group of degree $q$. Consequently $q$ is the largest prime dividing the order of $G$ and $B$ is a Sylow $q$-subgroup of $G$. Let $\mathcal{M}(A)$ be the set of all maximal subgroups of $A$ with index in $A$ not divisible by $p$. Since $A$ is not nilpotent, it is clear that $\mathcal{M}(A)$ is not empty. Consider $A_{1} \in \mathcal{M}(A)$. By hypothesis, $B A_{1}$ is a subgroup of $G$. Moreover the fact that $A \cap B=1$ implies $B A_{1}$ is a maximal subgroup of $G$. By Lemma 2.5 we obtain that $\operatorname{Core}_{G}\left(B A_{1}\right) \neq 1$ for all $A_{1} \in \mathcal{M}(A)$. In particular $N \leq B A_{1}$ for all $A_{1} \in \mathcal{M}(A)$. By Lemma 2.2, we have that $N \leq B\left[\bigcap\left\{A_{1} \mid A_{1} \in \mathcal{M}(A)\right\}\right]=B \Phi_{p}(A)$. Since $\Phi_{p}(A) \leq A$, it follows that it is supersoluble. In particular, $\Phi_{p}(A)$ is $p$-nilpotent. On the other hand, by Theorem 2.1, $\Phi_{p}(A)$ has a normal Sylow $p$-subgroup, $P$ say, such that $\Phi_{p}(A) / P$ is nilpotent. Hence $\Phi_{p}(A)$ is the direct product of two nilpotent subgroups and thus it is nilpotent. Since $|B|=q, B \Phi_{p}(A)$ is soluble by [8, VI, 4.3] and so $N$ is soluble, a contradiction.

Thus $\operatorname{Core}_{G}\left(A B_{1}\right) \neq 1$ for all maximal subgroups $B_{1}$ of $B$ (recall that $A$ permutes with every maximal subgroup of $B$ because $p$ does not divide $|B|)$. Furthermore, if Core $_{G}\left(B A_{1}\right)=1$ for some $A_{1} \in \mathcal{M}(A)$, it follows from Lemma 2.5 that $A$ has prime order, which contradicts that $A$ cannot be nilpotent, or $N \leq A$, which contradicts that $N$ is not soluble. So we may assume $\operatorname{Core}_{G}\left(B A_{1}\right) \neq 1$ for all $A_{1} \in \mathcal{M}(A)$. Arguing as above and applying again Lemma 2.2, we obtain that $N \leq A \Phi(B) \cap B \Phi_{p}(A)=$ $\Phi(B) \Phi_{p}(A)$. As above, $\Phi_{p}(A)$ is nilpotent. Then, by [8, VI, 4.3], $\Phi(B) \Phi_{p}(A)$ is soluble and so $N$ is soluble, a contradiction. Consequently $p$ divides the order of $A$ and $B$. 
Now we prove that $A$ and $B$ are non-nilpotent subgroups of $G$. Suppose that $A$ is nilpotent. If $B$ is nilpotent, then $G$ would be soluble by [8, VI, 4.3], a contradiction. Thus we may assume $B$ is not nilpotent and, in particular, that the number of distinct prime numbers dividing the order of $B$ is at least two. Moreover, $p$ divides the order of $B$ as we have proved before. Consider $B_{1} \in \mathcal{M}(B)$. By hypothesis and the fact that $A \cap B=1$, we have that $A B_{1}$ is a maximal subgroup of $G$. Arguing as above, we obtain that if $\operatorname{Core}_{G}\left(A B_{1}\right)=1$, then $B$ has prime order, against the fact that $B$ is not nilpotent, or $N \leq B$, which contradicts that $N$ is not soluble. Consequently we may assume $N \leq$ Core $_{G}\left(A B_{1}\right)$ for every $B_{1} \in \mathcal{M}(B)$. With analogous arguments to the ones used before we have $N \leq A \Phi_{p}(B)$ and $\Phi_{p}(B)$ is nilpotent. Therefore $N$ is soluble, a contradiction. Hence $A$ and similarly $B$ are non-nilpotent subgroups of $G$.

Finally note that if $N$ is contained in all maximal subgroups of $G$ of the form $A B_{1}$ and $B A_{1}$, where $B_{1} \in \mathcal{M}(B)$ and $A_{1} \in \mathcal{M}(A)$ (the fact that $A$ and $B$ are nonnilpotent yields that $\mathcal{M}(B)$ and $\mathcal{M}(A)$ are non-empty sets), then we would obtain $N \leq A \Phi_{p}(B) \cap B \Phi_{p}(A)=\Phi_{p}(A) \Phi_{p}(B)$. But $\Phi_{p}(A) \Phi_{p}(B)$ is a product of two nilpotent groups by the above arguments. Hence $N$ is soluble by [8, VI, 4.3], a contradiction. Consequently we may assume that there exists $A_{1} \in \mathcal{M}(A)$ such that $B A_{1}$ is a maximal subgroup of $G$ with $\operatorname{Core}_{G}\left(B A_{1}\right)=1$. Now Lemma 2.5 implies $A$ has prime order or $N \leq A$, the final contradiction.

Proof of Theorem 1.6. Assume that the result is false and let $G$ be a counterexample of minimal order. We argue that every proper quotient $G / N$, where $1 \neq N$ is a normal subgroup of $G$, is soluble. If $p$ does not divide $|G: N|$, then $G / N$ is soluble by the Feit-Thompson theorem. If $p$ divides $|G: N|$, it is clear that the hypotheses of the theorem hold in $G / N$. By minimality of $G$, we have that $G / N$ is soluble. Consequently, $\operatorname{Soc}(G)=N$ is the unique minimal normal subgroup of $G, N$ is non-abelian, and $\mathrm{C}_{G}(N)=1$. Arguing as in Theorem 1.5, we obtain that $A \cap B$ permutes with every maximal subgroup of $A$. Moreover, since $B$ is nilpotent, every maximal subgroup of $B$ is a normal subgroup of $B$. Hence $A \cap B$ permutes with every maximal subgroup of $B$. Now a similar argument to the one in used Theorem 1.5 yields $A \cap B=1$.

Note that $A$ is not nilpotent, otherwise $G$ would be soluble by [8, VI, 4.3], which contradicts the choice of $G$. Furthermore if $p$ does not divide the order of $A$, then $B$ permutes with every maximal subgroup of $A$ and so $G$ is soluble by [2, Theorem 1], against the choice of $G$. Thus we may assume $A$ is not nilpotent and $p$ divides the order of $A$. Consider now the set $\mathcal{M}(A)$ of all maximal subgroups of $A$ whose index in $A$ is not divisible by $p$. Since $A$ is not nilpotent, we have that $\mathcal{M}(A)$ is not empty. If $N$ is contained in $B A_{1}$ for all $A_{1} \in \mathcal{M}(A)$, then arguing as in Theorem 1.5 we obtain that $N \leq B \Phi_{p}(A)$ and $\Phi_{p}(A)$ is nilpotent. Thus by [8, VI, 4.3] $N$ is soluble, against the choice of $G$. Therefore there exists a maximal subgroup $A_{1}$ of $A$ such that $B A_{1}$ is a maximal subgroup of $G$ with $\operatorname{Core}_{G}\left(B A_{1}\right)=1$. By Lemma 2.5, $A$ has prime order or $N \leq A$, the final contradiction. 


\section{Final remarks}

The result of Theorem 1.4 does not hold when $p$ is not the smallest prime dividing the order of $G$. For instance, the alternating group $G=A_{5}$ of degree 5 can be expressed as the product of a cyclic group $A \cong C_{5}$ of order 5 the alternating group $B=A_{4}$ of degree 4 . In this case, $\operatorname{gcd}(|A|,|B|)=1,5$ divides $|A|$, and $B$ permutes with the unique maximal subgroup of $A$, but $G$ is not soluble.

In Theorem 1.6, the nilpotency of $B$ is required for the proof. The group $G=$ $\mathrm{PSL}_{2}$ (7) can be factorised as the product of a dihedral group $A$ of order 8 and nonabelian group $B$ of order 21 . Trivially, $B$ permutes with every maximal subgroup $A_{1}$ of $A$ such that 2 does not divide $\left|A: A_{1}\right|$, but $G$ is not soluble.

We do not know whether the hypothesis on $p$ in Theorem 1.5 is necessary.

\section{References}

[1] B. Amberg, S. Franciosi, and F. de Giovanni. Products of groups. Oxford Mathematical Monographs (The Clarendon Press Oxford University Press, New York, 1992). Oxford Science Publications.

[2] A. Ballester-Bolinches, J. Cossey, and M. C. Pedraza-Aguilera. 'On products of finite supersoluble groups'. Comm. Algebra 29 (7) (2001), 3145-3152.

[3] A. Ballester-Bolinches, R. Esteban-Romero, and M. Asaad. Products of finite groups, de Gruyter Expositions in Mathematics, Volume 53 (Walter de Gruyter, Berlin, 2010).

[4] A. Ballester-Bolinches, X. Guo, and M. C. Pedraza-Aguilera. 'A note on m-permutable products of finite groups'. J. Group Theory 3 (4) (2000), 381-384.

[5] W. E. Deskins. 'On maximal subgroups'. Proc. Symp. in Pure Math. Amer. Math. Soc. 1 (1959), 100-104.

[6] K. Doerk and T. Hawkes. Finite Soluble Groups, De Gruyter Expositions in Mathematics, Volume 4 (Walter de Gruyter, Berlin, New York, 1992).

[7] L. M. Ezquerro and X. Soler-Escrivà. 'On mutually M-permutable products of finite groups'. Comm. Algebra 31 (4) (2003), 1949-1960.

[8] B. Huppert. Endliche Gruppen I, Grund. Math. Wiss., Volume 134 (Springer Verlag, Berlin, Heidelberg, New York, 1967).

[9] R. Maier. 'Zur Vertauschbarkeit und Subnormalität von Untergruppen'. Arch. Math. (Basel) 53 (1989), 110-120.

M. Asaad, Department of Mathematics, Faculty of Science, Cairo University, Giza, Egypt

e-mail: moasmo45@ hotmail.com

A. Ballester-Bolinches, Departament d'Àlgebra, Universitat de València, Dr. Moliner, 50, 46100 Burjassot, València, Spain

e-mail: Adolfo.Ballester@uv.es

R. Esteban-Romero, Institut Universitari de Matemàtica Pura i Aplicada. Universitat Politècnica de València, Camí de Vera, s/n, 46022 València, Spain

e-mail: resteban@mat.upv.es 